\title{
Comparativo dos softwares de gerenciamento de referências bibliográficas: Mendeley, EndNote e Zotero
}

\author{
Comparing the bibliographic management \\ softwares: Mendeley, EndNote and Zotero
}

\author{
Eduardo Kazumi YAMAKAWA' \\ Flávio Issao KUBOTA' \\ Fernanda Hansch BEUREN' \\ Lisiane SCALVENZI' \\ Paulo Augusto CAUCHICK MIGUEL ${ }^{2}$
}

\section{Resumo}

A elaboração de uma revisão bibliográfica confiável, a partir de trabalhos relevantes publicados anteriormente, é fundamental para evidenciar a originalidade e a contribuição científica dos trabalhos de pesquisa. Devido à grande quantidade de bases de dados e de publicações disponíveis, torna-se necessário utilizar ferramentas que auxiliem na gestão das referências bibliográficas de uma maneira fácil e padronizada. O objetivo deste artigo é examinar três softwares de gerenciamento bibliográfico utilizados com frequência por pesquisadores acadêmicos, são eles:Mendeley, EndNote e Zotero. Nesse sentido, buscou-se, em primeiro lugar, evidenciar seus principais benefícios e as possíveis dificuldades de utilização. Em segundo lugar, procurou-se comparar suas principais características por meio de uma pesquisa teórico-conceitual baseada em literatura especializada, o que permitiu utilizá-los e analisá-los de maneira crítica. Assim sendo, evidenciou-se as principais particularidades de cada software e foi elaborado um quadro comparativo entre os mesmos. Considerando as características analisadas nos três softwares, concluiu-se que todos, ao mesmo tempo em que facilitam o trabalho dos pesquisadores, possuem ferramentas que facilitam as buscas, a organização e a análise dos artigos.

Palavras-chave: Comparativo. EndNote. Gerenciamento bibliográfico. Mendeley. Referências Bibliográficas. Zotero.

\begin{abstract}
The development of a reliable literature review from relevant previously published studies is imperative to highlight the originality and scientific contributions of research. Due to the large amount of databases and publications available, we need ease-to-use tools that assist reference management in a standardized way. The purpose of this article was to examine three of the most frequently used bibliographic management softwares by academic researchers: Mendeley, EndNote, and Zotero. The authors sought to highlight the main benefits and difficulties in using the softwares and compared their main features by using a theoretical-conceptual

\footnotetext{
1 Universidade Federal de Santa Catarina, Programa de Pós-Graduação em Engenharia de Produção, Departamento de Engenharia de Produção. Florianópolis, SC, Brasil.

2 Universidade Federal de Santa Catarina, Departamento de Engenharia de Produção e Sistemas. Campus Trindade, Caixa Postal 476, Cidade Universitária, 88040-900, Florianópolis, SC, Brasil. Correspondência para/Correspondence to: P.A. CAUCHICK MIGUEL. E-mail:<paulo.cauchick@ufsc.br>.
}

Recebido em 7/1/2013, reapresentado em 24/7/2013 e aceito para publicação em 13/9/2013.
\end{abstract}


research-based literature as well as critically analyzing the softwares cited by the authors. As a result, it was possible to highlight the main features of each of the softwares and develop a comparative chart. Considering the characteristics of the three softwares analyzed, it was possible to conclude that all of them have tools that facilitate searching, organizing, and analyzing articles, which can facilitate the work of researchers who use these softwares.

Keywords: Comparison. EndNote. Bibliographic management. Mendeley. Bibliographic Reference. Zotero.

\section{Introdução}

As referências, as instruções e a tecnologia caminham juntas no campo de suporte à pesquisa. Independente do nível de qualificação do pesquisador (desde o estudante que escreve o primeiro trabalho de pesquisa até os cientistas com Prêmio Nobel) as fontes de citação são fundamentais para uma boa pesquisa (Hensley, 2011). Ainda, Mugnaini (2003) afirma que o acúmulo e o armazenamento de informações, obtidos por intermédio da evolução da informática, suscitaram a necessidade de uma eficaz organização para posterior recuperação. Nesse contexto, o aumento, nos últimos anos, do número de informações disponíveis no meio digital demonstra que os pesquisadores necessitam encontrar formas rápidas e eficientes para gerenciar esses dados. Dessa forma, Duong (2010) destaca que a utilização de programas computacionais - que auxiliem neste gerenciamento - tornou-se um fator importante para facilitar o trabalho de usuários que precisam, frequentemente, buscar dados na literatura. Consequentemente, diversos programas computacionais vêm sendo desenvolvidos para este fim.

Nesse sentido, uma maior facilidade de acesso às diversas fontes de conhecimento vem se consolidando, especialmente por meio da Web, dos portais e das bases de periódicos. Por conseguinte, torna-se necessário estabelecer alguns critérios para privilegiar o uso de referências mais confiáveis e cientificamente mais embasadas; uma vez que dentro desse universo de textos e publicações, uma quantia significativa possui ressalvas quanto à confiabilidade. Somado a isso, com o passar do tempo, a produção contínua e constante do conhecimento acaba por dificultar a tarefa do pesquisador, uma vez que o volume de publicações e conteúdos se amplia consideravelmente. Uma observação deve ser apresentada: os programas não garantem a qualidade dos textos e das publicações gerenciadas, pois quem decide o que será gerenciado pelos mesmos são os pesquisadores. Assim, a qualidade é definida por estes.
Dessa forma, este artigo apresenta três programas utilizados, atualmente, por diversos pesquisadores: o Mendeley, o EndNote e o Zotero. Esses softwares são utilizados para buscar e organizar sistematicamente os artigos disponíveis na literatura.

O objetivo do trabalho é analisar e destacar os principais benefícios e algumas das dificuldades identificadas durante a utilização dos softwares citados acima. Essa investigação, comparando o uso dos programas computacionais, descreve alguns passos utilizados durante uma busca e uma organização bibliográfica.

Salienta-se, ainda, que este artigo apresenta: 1) a busca e a organização bibliográfica particularizada para os três programas computacionais analisados; 2) os métodos e as técnicas de pesquisa adotadas, bem como os critérios e os dados comparativos entre os softwares; 3) os benefícios e as dificuldades de cada programa computacional e a discussão dos resultados, conclusões e trabalhos futuros.

\section{Gerenciadores bibliográficos}

Uma revisão da literatura relevante é uma característica essencial de todo projeto acadêmico. Uma revisão efetiva cria uma sólida fundação para o avanço do conhecimento porque fecha áreas onde já existem muitas pesquisas e evidencia áreas onde pesquisas são necessárias (Webster \&Watsom, 2002). Diante da grande quantidade de dados que os pesquisadores necessitam para o desenvolvimento de alguma teoria e da quantidade de informações disponíveis, torna-se um desafio gerenciar de forma eficaz a quantidade de informação (Duong, 2010). Assim, muitos cientistas recorrem a programas computacionais que os auxiliem a buscar e a organizar bibliografias. Esses gerenciadores de referência bibliográfica arquivam e organizam os trabalhos que podem ser citados pelos pesquisadores. Registra-se, também, que entre as funções mais utilizadas estão as que obtêm informações de citação nas bases de dados 
online e aquelas que inserem essas informações de citação no documento num formato específico escolhido. Tal fato, automaticamente, gera uma referência bibliográfica completa no final do documento (Muldrow \&Yoder, 2009).

O Mendeley surgiu em 2008 e apresenta uma combinação de aplicação desktop e um Website. 0 desktop é instalado no computador do usuário. Já o Website serve para auxiliar pesquisadores no gerenciamento, compartilhamento e acesso a dados relacionados com determinada pesquisa. Esse software permite gerar estatísticas relacionadas ao número de artigos encontrados, regiões geográficas, identificação de leitores por área, autores que estão pesquisando sobre o tema de interesse, entre outros. O Mendeley trabalha com redes sociais; que possibilitam a interação entre a comunidade e os responsáveis pela ferramenta. Essa interação, sugere melhorias, quais sejam: a extração de metadados e a busca de textos em formato Portable Document Format (PDF) completos (Marchiori et al., 2010).

O EndNote teve sua primeira versão em 1988 para Macintosh. A versão atual pode ser utilizada nos ambientes desktop e Web. O EndNote desktop é um software que deve ser instalado no computador do usuário e serve para gerenciar referências bibliográficas. Esse serviço objetiva ser útil para pesquisar, armazenar e organizar as referências bibliográficas recolhidas em bases de dados credenciadas. O EndNote Web é similar ao desktop, pois também gerencia referências bibliográficas e é integrado à diversas bases de dados.

O Zotero foi lançado em 2006 numa versão Web. Na última versão pode ser utilizada uma combinação de aplicação desktop instalada no computador do usuário, e um aplicativo Web que é uma extensão do navegador de Internet Mozilla Firefox. O Zotero é desenvolvido pela Universidade de George Mason. Assim como o Mendeley e EndNote, o Zotero pode pesquisar, armazenar e organizar as referências bibliográficas obtidas em bases de dados credenciadas.

Dentro dessa diversidade de softwares, Fenner (2010) comenta que há uma proliferação de ferramentas de gestão de referência e estas estão sendo desenvolvidas para serem utilizadas não apenas em computadores, mas também em dispositivos móveis, quais sejam: telefones celulares e tablets. Os softwares de gerenciamento de referências são ferramentas que visam economizar tempo para os pesquisadores e, além disso, servem para agregar valor - independentemente de como estas ferramentas são exploradas (Zaugg et al., 2011). Quando o pesquisador utiliza essłe tipo de programa para organizar seu trabalho, possui mais tempo disponível para leitura e para escrita. Assim, além de organizar seus dados, ele pode identificar os trabalhos mais citados, os autores que mais escrevem sobre determinado assunto, os periódicos com maior número de publicações sobre determinado tema e outras formas de análises (e.g. análise das referências em cada publicação). Com esses dados, os pesquisadores podem ampliar o trabalho de pesquisa com informações que se destacam na sua área de atuação, pois conhecerão onde estão as informações mais relevantes, a partir de uma análise facilitada do grande volume de trabalhos existentes, escritos nos últimos anos.

Nesse sentido, Rokni et al. (2010) evidenciam que os autores que utilizam softwares gerenciadores de referência bibliográfica para escrever artigos científicos melhoram significativamente a qualidade dos manuscritos e ampliam o conhecimento sobre o tema que vem pesquisando. Ainda, Fenner (2010) destaca que os softwares de gerenciamento de referências auxiliam os pesquisadores através da realização de três funções básicas:

1) Busca: Iocalização de literatura científica relevante;

2) Armazenamento: guardar os resultados da pesquisa em um banco de dados pessoal para futuro acesso e;

3) Escrita: inserir as referências quando estiver escrevendo um manuscrito.

Sombatsompop et al. (2007) apresentam a importância de utilizar um software de gerenciamento bibliográfico para buscar informações contidas nos artigos da área de interesse de cada pesquisador. Essa atitude procura facilitar o trabalho, pois além de trazer os arquivos dos artigos das bases de dados para o software, trazem também dados importantes: nomes completos dos autores dos artigos, periódicos onde os mesmos são publicados, títulos, resumos e outras informações pertinentes numa revisão bibliográfica e análise bibliométrica. 


\section{Métodos}

Delineou-se este estudo a partir da pesquisa teórico-conceitual (Berto \& Nakano, 2000; Miguel, 2010), baseada na literatura e na utilização pelos autores dos softwares de busca e organização bibliográfica:Mendeley, EndNote e Zotero. As bases de dados utilizadas para a busca de artigos na literatura foram a Scopus e Web of Science, as quais contêm diversos periódicos importantes para a área de atuação destes pesquisadores, que é a Engenharia de Produção. A busca foi realizada a partir das palavras-chave:"software Endnote", "software Zotero"e "software Mendeley". Foram encontrados 28 artigos que tratam das palavras-chave apresentadas. Esses artigos foram lidos na íntegra a fim de identificar o que a literatura vem tratando sobre cada software.

O objetivo da pesquisa é apresentar a comparação entre os softwares citados apresentando o que cada um traz em termos de benefícios e dificuldades utilização.

Os dados foram obtidos por meio de informações coletadas a partir do uso destes programas pelos autores (Figura 1). A análise dos dados coletados foi tabulada para assim apresentar, de forma sistemática, os benefícios e dificuldades identificadas durante a utilização de cada um dos softwares. Primeiramente, descreve-se cada software em separado para, posteriormente, realizar uma síntese comparativa entre eles no final dos resultados. Na avaliação comparativa entre os três programas computacionais, foram consideradas as seguintes características de uso: disponibilidade (aquisição do software), sincronização de arquivos (desktop e Web), monitoramento automático de diretórios, visualização de referências duplicadas, interação com a Web, inserção prática de citações nos textos, disponibilidade dos programas para diversos sistemas operacionais, facilidade na leitura dos arquivos recuperados, disponibilidade dos dados básicos de artigos (palavras-chave, periódico, autores etc.), busca em base de dados e possibilidade de importação e exportação de dados.

\section{Características e funções dos gerenciadores}

Esta seção apresenta os benefícios e dificuldades encontradas durante o uso dos softwares de gerenciamento bibliográfico.

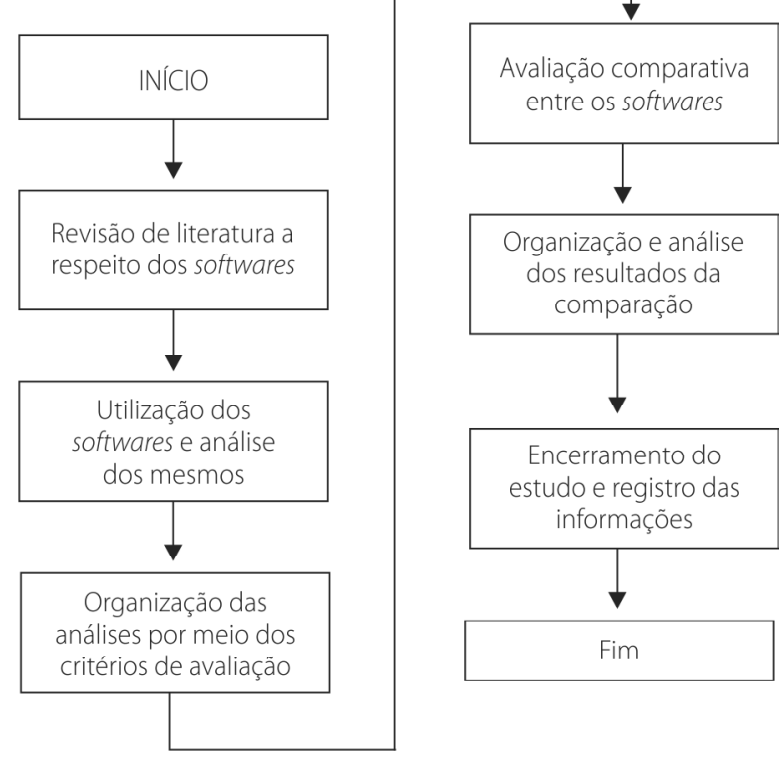

Figura 1. Etapas do método de pesquisa.

Fonte: Elaborada pelos autores (2012).

\section{Mendeley (versão 1.5.2)}

O Mendeley pode ser utilizado por meio de um aplicativo instalado no computador chamado "Mendeley Desktop" ou pelo acesso ao site do mesmo <http:// www.mendeley.com>. O aplicativo não possui custo de aquisição. Após a utilização e interação com o software Mendeley, foi possível obter informações a respeito de aspectos de vantagens e desvantagens do programa. Essas características são descritas nas subseções seguintes.

O primeiro benefício encontrado diz respeito à sincronização dos arquivos armazenados no programa. Com o Mendeley, é possível ter os arquivos de trabalho gravados tanto nos computadores de uso pessoal quanto na rede (Internet). Assim, pode-se acessar os mesmos arquivos em rede, o que gera vantagens tanto em segurança (eventuais perdas) quanto em acessibilidade dos dados. Caso o usuário não esteja satisfeito com essas disponibilidades, o programa também permite a realização de cópia manual de segurança, com o objetivo de garantir mais um backup de arquivos.

Além disso, com o Mendeley, também, é possível o monitoramento automático dos diretórios utilizados 
para armazenagem dos arquivos. Ou seja, é possível manter monitorada uma pasta, na qual são inseridos todos os arquivos; assim, o Mendeley, automaticamente, grava-os na pasta de armazenamento. Também, é possível realizar anotações e marcações pessoais nos próprios arquivos. Ainda no ambiente online na Web, a conta de usuário possui espaço gratuito de 1GB para armazenamento de documentos diversos. Em casa de necessidade, é possível aderir aos planos da empresa responsável pelo Mendeley, conforme a quantidade de espaço a mais desejada. Uma observação importante é que a sincronização web precisa, obrigatoriamente, estar ativa no Mendeley Desktop com seu login e senha configurados para que a cópia seja realizada no ambiente Web.

Um dos pontos positivos de utilização do Mendeley é a facilidade de acesso. É possível acessar a biblioteca de dados em qualquer lugar que possua conexão com a internet, com ou sem o Mendeley Desktop instalado. No caso de utilizar o Mendeley Desktop, o mesmo pode ser instalado em vários computadores diferentes podendo ser acessado de vários lugares diferentes e com o mesmo conteúdo, desde que haja acesso a Internet para realizar a sincronização com o conteúdo na sua conta Web. No caso de não ter o Mendeley Desktop instalado, é possível realizar o acesso pela conta pessoal no site do software com toda a sua biblioteca, previamente sincronizada, disponível. Além disso, também é possível realizar o acesso à biblioteca pessoal pelo aplicativo móvel "Mendeley - Reference Manager (Lite)" para uso em aparelhos celulares móveis que possuem recursos para navegação.

Outro benefício percebido é a facilidade em obter os dados completos a respeito do artigo, tais como os autores, periódico publicado, volume, número etc. Isso pode ser feito por meio da inserção do Digital Object Identifier (DOI). No entanto, observou-se que, em alguns casos, o preenchimento do DOI não foi suficiente, ou seja, alguns dados estavam incompletos. Em específico, durante a análise, o principal problema encontrado relaciona-se com os dados referentes aos autores dos trabalhos: apenas o primeiro autor aparecia, sendo que os artigos possuíam dois ou mais autores.

Acerca do compartilhamento de informações, o programa permite sincronizar arquivos entre os usuários do software. Esse aspecto é benéfico por diversos fatores, dentre os quais o compartilhamento de trabalhos entre autores que pesquisam sobre um mesmo tema e/ou entre integrantes de grupo de estudos em determinado assunto, por exemplo. Além disso, o usuário pode gerenciar e selecionar os arquivos que deseja compartilhar com os demais pesquisadores/usuários do Mendeley. Junto a isso, o software também permite a sincronização e a criação de grupos online, com sincronização entre computadores e Web. Dentro desses grupos é possível subdividir em vários diretórios (pastas) para separar assuntos para melhor estruturar uma pesquisa.

Outro aspecto encontrado é a possibilidade de inserir citações de forma prática nos arquivos de texto, bem como realizar a mescla dessas (em caso de duas ou mais citações para um determinado conceito, por exemplo). Simultaneamente às citações, ocorrem nesta ação as inserções das respectivas referências dentro das formatações desejadas: Associação Brasileira de Normas Técnicas (ABNT) ou American Psychological Association (APA), por exemplo; ou, até mesmo, outra. Depois de finalizada a inserção das referências, é possível salvar uma versão do arquivo sem vínculos com o Mendeley (isso é, as referências ficam conectadas ao arquivo). Nesse aspecto, ressalta-se ainda a possibilidade de importação de diversos estilos de referências (numeradas, nominais etc.).

O programa também facilita o gerenciamento dos dados dos arquivos armazenados. Na aba "Needs Review" (Necessita Revisão) se encontram os documentos que precisam ter seus dados atualizados e a aba"Recently Added" (Recentemente Adicionado) apresenta os arquivos adicionados mais recentemente. Por fim, a aba "Favorites" (Favoritos) pode ser utilizada para separar os trabalhos preferenciais para uso em determinado estudo e/ou pesquisa. É importante ressaltar que essas abas estão disponíveis tanto na versão desktop (uso no próprio computador) do programa quanto na Web (conta de acesso do usuário), podendo ser sincronizadas.

Acerca dos Sistemas Operacionais (SO), o Mendeley, assim como o Zotero, possui suporte para três: Windows, Mac e Linux. Referente aos aspectos de acessibilidade do software, verificou-se que o mesmo possui versões e aplicativos disponíveis para diversos recursos; além de permitir o acesso a partir de computadores, tablets, celulares etc. 
Quanto à utilização na Web, por meio da utilização do Mendeley é possível encontrar pessoas que pesquisam temas semelhantes e compartilhar artigos que possam ser relevantes aos outros pesquisadores. Ainda, é possível visualizar as ações das demais pessoas incluídas na rede de contatos por meio dos feeds (atualizações em tempo real), ou seja, é uma espécie de "rede social acadêmica" que funciona via Web. No caso do Mendeley Desktop, é possível somente atualizar os feeds e adicionar novas pessoas para um determinado grupo.

Os arquivos no Mendeley podem ser acessados e lidos de forma ampla, sem a necessidade de ajustes no layout do programa.

No entanto, durante a utilização deste software, algumas dificuldades também foram encontradas, principalmente em aspectos funcionais. Uma das principais dificuldades encontradas na utilização do Mendeley é com relação aos dados bibliométricos mais simples, por exemplo, a quantidade de autores, de palavras-chave, de ano de publicação etc. O programa não possui mecanismos que possam realizar a contagem desses dados, o que poderia facilitar consideravelmente o trabalho dos usuários envolvendo análise sistemática da literatura por meio da bibliometria.

Outra desvantagem encontrada é que o software não permite realizar buscas em bases de dados dentro de sua própria estrutura. No entanto, segundo a empresa responsável pelo programa, esforços já estão sendo direcionados para a viabilização dessa característica.

Em relação à criação de grupos e compartilhamentos, o programa somente possibilita criar 5 grupos privados, com no máximo 10 integrantes na conta padrão, a free (grátis). Caso ocorra a necessidade de números superiores aos mencionados, o usuário deverá escolher os planos disponíveis e pagar pelos mesmos. Apesar de possuir diversas formas de acesso, não existe um aplicativo móvel oficial para uso com sistemas Android. Existem alguns aplicativos não oficiais que podem ser utilizados, no entanto, possuem vários problemas na utilização e instalação.

Quanto à funcionalidade nas citações das referências, percebeu-se que o Mendeley não permite a alteração dinâmica na formatação das citações nos textos. Sendo assim, não é possível alterar, quando necessário, a maneira de citar os autores (exemplo: de "Autor (2012)" para "(AUTOR, 2012)"). Ainda em relação à funcionalidade, mas na perspectiva de busca por artigos, o recurso "Import" do Mendeley não funciona em determinados navegadores, como é o caso do Internet Explorer.

\section{EndNote (versão X5)}

O EndNote é um software de gerenciamento de referências bibliográficas, com licença paga, bastante utilizado nos ambientes onde se realiza a pesquisa acadêmica. O EndNote inclui o EndNote Web, que é uma ferramenta baseada em internet que fornece um ambiente colaborativo online com a possibilidade de organizar listas de publicação nas comunidades do autor.

O EndNote possibilita salvar os arquivos de trabalho tanto nos computadores de uso pessoal quanto na rede (Internet) por meio do EndNote Web. Porém, para que isso seja possível, é necessário que o usuário tenha uma conta de acesso no site da empresa responsável pelo software e, para isso, o mesmo também deve adquirir uma licença válida do programa EndNote, que é paga. Apesar de não possuir todas as funcionalidades do EndNote Desktop, o EndNote Web possui algumas vantagens na integração com as bases de dados da empresa Thomson Reuters, como, por exemplo, a ISI Web of Knowledge, onde é possível recolher dados de registros relacionados e número de citações de maneira integrada ao próprio sistema.

Com o EndNote é possível encontrar textos completos de artigos automaticamente e baixá-los utilizando a opção "Find full text". Quando o texto completo é localizado, o EndNote baixa o arquivo e adiciona o link à referência automaticamente. Possibilita também, a busca por referências bibliográficas já arquivadas no computador do usuário a partir da seleção dos arquivos que são arrastados para o campo "PDF\& Quick Edit". Entretanto, o computador deve estar conectado à Internet e os artigos devem estar cadastrados nas bases de dados que têm acesso ao programa.

O software EndNote também apresenta outro benefício, a visualização de referências duplicadas, onde se pode escolher as opções que apresentam o maior número de informações, onde é possível selecionar a versão que deve ser mantida e qual deve ser apagada. $\bigcirc$ 
comando "Cite while you write", também é um benefício deste software, o qual seleciona a referência que se deseja utilizar, podendo exportá-la para o documento que esteja sendo escrito nas plataformas Microsoft Word, Apple Pages '09, OpenOffice.org, Writer 3 ou Mathematica 8.

No EndNote é possível anexar até 45 arquivos por referência bibliográfica. Estes arquivos podem ser do tipo PDF, Word ou ainda figuras. Os arquivos com a extensão em PDF anexados no software podem ser utilizados não apenas para visualização do documento, mas também para anotações, comentários e destaques - é possível, ainda, realçar as partes do texto com uma ferramenta que cria um efeito similar à caneta marca textos.

A principal dificuldade encontrada pelo software EndNote é que a licença para sua utilização é paga, dificultando o acesso de diversos usuários. Cabe destacar que o EndNote deve ser atualizado frequentemente, gerando mais custos para sua utilização. Outra dificuldade encontrada por usuários desse programa, está relacionado com os sistemas operacionais (SOs), pois o EndNote possui suporte apenas para dois destes: o Windows e o Mac. Os usuários do sistema operacional Linux não possuem até o momento versão compatível do EndNote.

Uma limitação considerada pelos autores como a mais preocupante, está relacionada com as informações baixadas pelo EndNote. Esses dados são salvos no disco rígido do computador, incluindo os arquivos anexados, como os PDF dos artigos. Entretanto, estes dados permanecem apenas no computador do usuário, no qual existe o risco de perda ou extravio destas informações, as quais não são disponíveis online pelo EndNote Web. Outra situação relacionada com este arquivamento é o espaço necessário do computador para arquivar os dados.

\section{Zotero (versão 3.0.11)}

O Zotero pode ser utilizado por meio de um complemento instalado no navegador de Internet Mozilla Firefox ${ }^{\circledR}$ ou por meio do programa instalado no computador Zotero Standalone. O aplicativo não possui custo de aquisição.

O Zotero é um gerenciador de referência bibliográfica de código aberto, arquivador de documentos, gerenciador de citações e uma ferramenta de colabo- ração. A versão Zotero Web é um complemento do navegador Mozilla Firefox. A versão Zotero Standalone também está disponível para os navegadores de Internet Chrome e Safari. O Zotero é compatível com diversas bases de dados como ISI Web of Knowledge, Google Scholar, PubMed, entre diversos periódicos e bases de dados de universidades. Por meio de um clique no ícone de navegação localizada na barra do navegador as informações bibliográficas como autor, título, periódico, volume, número de páginas etc., são salvas criando um arquivo com todas as referências. As referências também podem ser inseridas manualmente.

O Zotero também pode importar os artigos em PDF existentes, além de outros tipos de arquivo como arquivos de imagem e vídeo. Para os arquivos PDF, além da visualização é possível destacar trechos e fazer anotações no arquivo dentro do Zotero. As citações podem ser adicionadas ao Microsoft Word, Open Office, Látex e GloogleDocs. Diversos estilos de citação estão disponíveis no repositório de estilos.

O Zotero fornece grátis até $100 \mathrm{MB}$ de espaço para armazenamento online, cópia de segurança e sincronização de referências e anexos permitindo que a base de dados das referências bibliográficas seja acessada a partir de diversos computadores. Existe, também, a possibilidade de sincronizar esses dados com os serviços de armazenamento na internet, como o iCloud, da Apple, os quais deverão suportar o protocolo WebDav. As referências podem ser compartilhadas com os colegas por meio de bibliotecas públicas e privadas.

Referente aos sistemas operacionais, o Zotero possui suporte para os sistemas operacionais Windows, Mac e Linux, sendo, assim, disponível para usuários de diversos sistemas operacionais.

O Zotero em sua versão atual ainda não é compatível com bases de dados como Scopus e Reaxsys. Para se salvar as informações das referências bibliográficas é necessário importar em algum formato compatível como Research Information Systems (RIS) e BibTeX. O programa não possui mecanismos que possa realizar a contagem de dados bibliométricos mais simples, por exemplo: a quantidade de autores, as palavras-chave, o ano de publicação etc. O software não permite realizar buscas em bases de dados dentro de sua própria estrutura. 
Em seguida, encerrando-se a seção de resultados, é apresentado um resumo comparativo dos três softwares analisados neste trabalho.

\section{Resumo comparativo entre Mendeley, EndNote e Zotero}

Nesta seção, realiza-se uma análise comparativa entre as características de cada um dos softwares estudados. Inicialmente, o Quadro 1 mostra, de maneira resumida, o comparativo de algumas propriedades do Mendeley, EndNote e Zotero.

Percebe-se que tanto o Mendeley quanto o Zotero são softwares gratuitos e, por isso, possuem uma melhor facilidade de acesso em relação ao EndNote. No que diz respeito à sincronização de arquivos, observou-se que o Mendeley é o programa mais vantajoso, uma vez que permite o armazenamento dos arquivos tanto no computador do usuário quanto na Web; fato este que possibilita salvar e sincronizar as alterações e marcações feitas nos artigos em estudo.

Em relação à visualização de referências duplicadas, notou-se que o EndNote é o software com mais recursos de praticidade e facilidade nesse aspecto, pois há opções específicas para detectar os trabalhos armazenados e duplicados (normalmente artigos vinculados à diversas bases de dados como Scopus, Compendex etc.). O Zotero também possui essa opção. Nesse quesito, o Mendeley é o programa menos eficiente. Acerca da interação com a Web, foi observado que os três programas de gerenciamento de referências possuem recursos para isso.

Na inserção de citações durante a redação de textos, o Mendeley é o software menos benéfico, uma vez que não possui opção prática para tal função, ao passo que o EndNote e o Zotero, além de permitir a inserção de citações, possuem, também, a disponibilidade para alterar os diversos tipos de citação. A respeito da disponibilidade para sistemas operacionais, o Mendeley possui uma pequena vantagem em relação ao Zotero. Ambos possuem versões para Windows, Mac e Linux. No entanto, o Mendeley possui aplicativo específico para usuários de celulares que possuem o sistema Android ("Mendeley Reference Manager (Lite)").
Na leitura de arquivos, os programas Mendeley e Zotero têm um layout mais amigável para essa atividade, sem a necessidade de ajuste na tela do software, diferente do que ocorre com o EndNote. Por outro lado, em relação à visualização e ao gerenciamento de dados básicos (tais como palavras-chave, autores, instituições dos autores etc.), o mesmo EndNote é o programa mais adequado, uma vez que consegue desenvolver uma contagem mais prática e automática, fornecendo esses dados de forma mais rápida e precisa do que o Mendeley e o Zotero. Por fim, na busca em base de dados, o EndNote é o único software que possibilita acessar algumas bases dentro de sua própria estrutura no desktop, dentre as quais a EBSCO e a Web of Science, por exemplo.

\section{Conclusão}

O objetivo do presente trabalho foi destacar os benefícios e as dificuldades de utilização dos softwares Mendeley, EndNote e Zotero, empregados para realizar busca de referências bibliográficas na literatura e, ao mesmo tempo, fazer sua organização. Foram descritos alguns passos normalmente utilizados durante a pesquisa de comparação dos três softwares. Analisando os três programas, foi possível identificar as principais características de cada software, seus benefícios e dificuldades - além de visualizar em quais contextos e situações um programa se destaca em relação ao outro.

Quanto à facilidade de obtenção e utilização, o Mendeley e o Zotero se destacaram, principalmente, devido ao fato de esses serem gratuitos e com recursos eficientes no gerenciamento bibliográfico, bem como a maior disponibilidade para diferentes sistemas operacionais. Além disso, o Mendeley e o Zotero permitem o armazenamento simultâneo dos arquivos no disco rígido do computador e na Web, por meio da conta de acesso do usuário. Ainda, os três programas organizam dados básicos dos trabalhos organizados depois de feitas as buscas (palavras-chave, periódico, autores etc.). No entanto, foi possível observar que o EndNote possui maior facilidade de operação para contagem e sistematização dessas informações, o que facilita a realização de análises bibliométricas, tais como, por exemplo: os autores que mais se destacam em determinado tema de pesquisa, nos periódicos de referência, nas palavras-chave mais utilizadas, nos autores mais citados etc. 
Quadro 1. Resumo comparativo dos softwares Mendeley, EndNote e Zotero.

\begin{tabular}{|c|c|c|}
\hline Características & Mendeley & EndNote \\
\hline Disponibilidade & Software livre & Software pago \\
\hline $\begin{array}{l}\text { Sincronização de arquivos } \\
\text { (desktop e Web) }\end{array}$ & $\begin{array}{l}\text { Existente. Principal benefício: } \\
\text { backup de arquivos na Web, em } \\
\text { caso de problemas com o compu- } \\
\text { tador pessoal do usuário. }\end{array}$ & $\begin{array}{l}\text { Existente. Até } 25 \text { mil referências e } \\
\text { 1GB de espaço para anexos. }\end{array}$ \\
\hline $\begin{array}{l}\text { Monitoramento automático } \\
\text { de diretórios }\end{array}$ & $\begin{array}{l}\text { Existente. As alterações realiza- } \\
\text { das em determinado arquivo são } \\
\text { transferidas para a versão salva na } \\
\text { Web (ou desktop, em caso das } \\
\text { alterações terem sido feitas na } \\
\text { Web). }\end{array}$ & $\begin{array}{l}\text { As alterações não são realizadas } \\
\text { de maneira automática. }\end{array}$ \\
\hline $\begin{array}{l}\text { Visualização de referências } \\
\text { duplicadas }\end{array}$ & $\begin{array}{l}\text { Não há mecanismo específico } \\
\text { para essa atividade. O que se } \\
\text { visualiza no Mendeleysão arquivos } \\
\text { que necessitam ter seus dados } \\
\text { revisados(comando "Needsreview"). }\end{array}$ & $\begin{array}{l}\text { Fácil visualização por meio do } \\
\text { comando "Find Duplicates", que } \\
\text { encontra as referências repetidas } \\
\text { e possibilita eliminá-las do dire- } \\
\text { tório de arquivos. }\end{array}$ \\
\hline
\end{tabular}

Interação com a Web

Inserção prática de citações nos textos

Sistemas operacionais (versões disponíveis)

Leitura de arquivos

Dados básicos de artigos (palavras-chave, periódico, autores etc.)

Busca em base de dados

Possibilidade de importação de dados

Possibilidade de exportação de dados
Possibilidade de armazenar e Possibilidade de armazenar e compartilhar arquivos na rede, compartilhar arquivos na rede, bem como interagir com outros bem como interagir com outros usuários do software. usuários do software.

Existente por meio do comando Existente por meio da aba "Insert "Alt+M" no editor de textos. Citation" (criada no editor de Limitação: não permite alteração textos após a instalação do prática na forma de citação, por EndNote) e mais efetiva, pois perexemplo: de "Autor (2012)" para mite a alteração que é restrita no "(AUTOR, 2012)". caso do Mendeley.

Windows, Mac e Linux, além de Windows e Mac. Não há versão um aplicativo para celulares para Linux.

("Mendeley - Reference Manager $\left.(\text { Lite })^{\prime \prime}\right)$.

Fácil e prática, pois não necessita Necessidade de ajustes no layout a abertura de arquivos pdf com do programa o que dificulta a softwares específicos. O próprio leitura dos arquivos, porém o Mendeley oferece a estrutura para próprio EndNote oferece estrutura a leitura para leitura

Contagem "mecânica", o que Contagem mais prática eautomádificulta a elaboração de levanta- tica. Fornece os mesmos dados mento de dados bibliométricos de forma rápida e precisa. simples com o uso do Mendeley.

Não permite realizar busca de É possível realizar busca em algudados dentro de sua estrutura

mas bases de dados por meio do próprio EndNote (Ex: EBSCO, Web of Science, PubMed etc.).

Sim. Formatos: BibTex, RIS, EndNote Sim. Formatos: Txt, Rtf, Htm eXML $X M L$ e Zotero Library

Sim. Formatos: BibTex, RIS e Sim. Formatos: EndNote XML, BiEndNote XML blioteca EndNote, Refer/Bib/X, Tab Delimited, RIS, ISI-CE e Multi-filter
Possibilidade de armazenar e compartilhar arquivos na rede, bem como interagir com outros usuários do software.

Existente por meio da opção "Copiar Citação" no menu Editar. É possível escolher entre diversos estilos de citação.

Windows, Mac e Linux

Fácil e prática, pois não necessita a abertura de arquivos pdf com softwares específicos. O próprio Zotero oferece a estrutura para a leitura

Contagem "mecânica", o que dificulta a elaboração de levantamento de dados bibliométricos simples com o uso do Zotero

Não permite realizar busca de dados dentro de sua estrutura

Sim. Formatos: Zotero RDF, MODS, BibTex, Refer/BibIX, Unqualified Dublin Core RDF

Sim. Formatos: Bibliontology, RDF, BibTex, Bookmarks, Coins, MODS, Refer/BibIX, RIS, TEI, Unqualified Dublin Core RDF, Wikipedia Citation Templates e Zotero RDF

Fonte: Elaborado pelos autores (2012). 
Após a utilização dos softwares analisados, pôde-se concluir que todos apresentam ferramentas que facilitam as buscas e análises de artigos, principalmente, em revisões bibliométricas da literatura em diferentes áreas. Uma limitação do trabalho diz respeito à análise dos softwares estudados. Essa análise foi executada pelos autores deste trabalho fazendo com que as considerações do presente artigo se restrinjam a esses pontos de vista. Além disso, não é função deste estudo o julgamento de qual, dentre os softwares analisados, é o mais adequado para ser utilizados pela comunidade acadêmica. No entanto, os resultados e descobertas descritas são úteis para melhor visualizar os benefícios e dificuldades de cada programa e orientar para a tomada de decisão particular dos pesquisadores a respeito de qual software utilizar.

Por fim, para oportunidade futura, sugere-se o desenvolvimento de uma pesquisa baseada numa survey ou entrevistas semiestruturadas (aspecto a ser melhor definido) com usuários de softwares de gerenciamento bibliográfico. Tal investigação visará uma análise mais aprofundada das características, das necessidades e das preferências de pesquisadores e usuários dessas ferramentas.

\section{Referências}

Berto, R.M.V.S.; Nakano, D.N. A produção científica nos anais do Encontro Nacional de Engenharia de Produção: um levantamento de métodos e tipos de pesquisa. Produção, v.9, n.2, p.65-76, 2000.

Duong, K. Rolling out Zotero across campus as a part of a science librarian's outreach efforts. Science and Technology Libraries, v.29, n.4, p.315-324, 2010.

Fenner, M. Reference management meets Web 2.0. Cellular Therapy and Transplantation, v.2, n.6, p.1-3, 2010.

Hensley, M.K. Citation management software: Features and futures. Reference \& User Services Quarterly, v.50, n.3, p.204-208, 2011.

Marchiori, P.Z. et al. Aspectos estruturais e motivacionais e possíveis zoneamentos discursivos em software social acadêmico. Liinc em Revista, v.6, n.2, p.355-369, 2010.

Miguel, P.A.C. (Org.). Metodologia de pesquisa em engenharia de produção e gestão de operações. Rio de Janeiro: Elsevier, 2010.
Mugnaini, R. A bibliometria na exploração de bases de dados: a importância da Linguística. Transinformação, v.15, n.1, p.45-52, 2003.

Muldrow, J.; Yoder, S. Out of cite! how reference managers are taking research to the next level. Political Science \& Politics, v.42, n.1, p.167-172, 2009.

Rokni, L. et al. A comparative analysis of writing scientific references manually and by using endnote bibliographic software. Pakistan Journal of Medical Sciences, v.26, n.1, p.229-232, 2010.

Sombatsompop, N. et al. Categorization and trend of materials science research from Science Citation Index (SCI) database: A case study of ceramics, metallurgy, and polymer subfields. Scientometrics, v.71, n.2, p.283-302, 2007.

Webster, J.; Watsom, R.T. Analyzing the past to prepare for the future: Writing a literature review. Management Information Systems Quarterly, v.26, n.2, p.13-23, 2002.

Zaugg, H. et al. Mendeley: Creating communities of scholarly inquiry through research collaboration. TechTrends, v.55, n.1, p.32-36, 2011. 
